\title{
On the associated primes of Matlis duals of local cohomology modules II
}

\author{
Michael Hellus
}

May 29, 2022

\begin{abstract}
In continuation of [1] we study associated primes of Matlis duals of local cohomology modules (MDLCM). We combine ideas from Helmut Zöschinger on coassociated primes of arbitrary modules with results from [1], 4], [5], 6] and obtain partial answers to questions which were left open in [1]. These partial answers give further support for conjecture $(*)$ from [1] on the set of associated primes of MDLCMs. In addition, and also inspired by ideas from Zöschinger, we prove some non-finiteness results of local cohomology.
\end{abstract}

\section{Introduction}

Let $I$ be an ideal of a local, noetherian ring $R$. By $H_{I}^{l}$ we denote the $l$-th local cohomology functor supported on $I$, by $E$ a fixed $R$-injective hull of the residue field of $R$ and by $D$ the Matlis dual functor $D:=\operatorname{Hom}_{R}(\ldots, E)$ from $(R-\bmod )$ to $(R-\bmod )$.

Suppose that one has $H_{I}^{l}(R)=0$ for $l \neq c$ ( $c$ is necessarily the height of $I$ then). Assume that a regular sequence $x_{1}, \ldots, x_{c}$ in $I$ is given. It was shown in the author's Habilitationsschrift ([2, Cor. 1.1.4]) that $I$ is a settheoretic complete intersection defined by the $x_{i}$ if and only if the $x_{i}$ form a $D\left(H_{I}^{c}(R)\right.$ )-(quasi)regular sequence. This gives strong motivation to study the associated primes of $D\left(H_{I}^{c}(R)\right)$. It is this study which we started in [1] and which we continue here.

The simplest case is $R=k\left[\left[X_{1}, \ldots, X_{n}\right]\right]$ and $I=\left(X_{1}, \ldots, X_{c}\right) R$, where $k$ is a field, the $X_{i}$ are indeterminates and $0 \leq c \leq n$. The case $c=n$ is easy; the case $c=n-1$ is non-trivial and was completely solved in [5, Theorem 2.5], see also [1]. The next case is $c=n-2$, where the following is known ([1, Theorem 2.2.1] and [5, Theorem 1.3(ii),(v)]):

$$
p \in \operatorname{Ass}_{R}\left(D\left(H_{\left(X_{1}, \ldots, X_{n-2}\right)}^{n-2}(R)\right)\right) \Rightarrow \text { height } p \in\{0,1,2\}
$$




$$
\{0\} \in \operatorname{Ass}_{R}\left(D\left(H_{\left(X_{1}, \ldots, X_{n-2}\right)}^{n-2}(R)\right)\right) .
$$

- If height $p=2$ :

$$
p \in \operatorname{Ass}_{R}\left(D\left(H_{\left(X_{1}, \ldots, X_{n-2}\right)}^{n-2}(R)\right)\right) \Longleftrightarrow \sqrt{p+\left(X_{1}, \ldots, X_{n-2}\right)}=\sqrt{\left(X_{1}, \ldots X_{n}\right)}
$$

- If height $p=1: P$ is generated by a prime element $p$ of $R: P=p R$. If $p \notin\left(X_{1}, \ldots, X_{n-2}\right)$, then

$$
p R \in \operatorname{Ass}_{R}\left(D\left(H_{\left(X_{1}, \ldots, X_{n-2}\right)}^{n-2}(R)\right)\right) .
$$

It is natural to ask next

Question 1.1. Which height-one prime ideals, i. e. which $P=p R$, where $p$ is an (arbitrary) prime element of $R$, are in $\operatorname{Ass}_{R}(\underbrace{D\left(H_{\left(X_{1}, \ldots, X_{n}-2\right)}^{n-2}(R)\right)}_{=: D})$ ?

This question is open (but note that some very special height one prime ideals in $\operatorname{Ass}_{R}(D)$ where found in [2, Cor. 4.3.1]). The main goal of this paper is to show that in many cases the answer to question 1.1 is positive; in particular, it is positive if $k$ is countable and $p$ is a polynomial contained in $\left(X_{n-1}, X_{n}\right) R$. In fact, our two main results, theorem 2.1 and theorem 2.2, are both a little more general, see section 2 for the precise statements. An example which is by no means trivial and where question 1.1 has a positive answer is given by $p=X_{n-1} X_{1}+X_{n} X_{2}$ (if $n \geq 4$, of course). This example follows from theorem 2.2.

The results in section 2 give some indication that conjecture $(*)$ from [1, section 1] (which says in this situation that

$$
\operatorname{Ass}_{R}(D)=\left\{p \mid H_{\left(X_{1}, \ldots, X_{n-2}\right)}^{n-2}(R / p) \neq 0\right\}
$$

) holds, because in the situation of theorem 2.1 one has $H_{I}^{n-2}(R /(a, b) R) \neq 0$ and, a fortiori, $H_{I}^{n-2}(R / p R) \neq 0$; in this context, see also [1, Theorem 1.1].

In section 3 we prove some non-finiteness properties of local cohomology modules: It is very well-known that top local cohomology modules are almost never finitely generated, see e. g. [3, Remark 2.5] for a quick proof using the Nakayama lemma. In fact a stronger statement holds: No quotient of a top local cohomology module is finite (corollary 3.3), and we do not even have to assume that the module $M$ whose local cohomology we consider must be finite. One even has that top local cohomology modules have no coatomic quotients (theorem 3.2, a module is coatomic if every proper submodule is contained in a maximal one).

Helmut Zöschinger's work on coatomic modules and coassociated prime ideals (e. g., [7], [8], [9]) is essential for both sections of this paper. 


\section{Associated prime ideals}

By 'countable' we shall mean either finite or 'infinite countable'.

Theorem 2.1. Let $k$ be a countable field, $R$ a domain and a local $k$-algebra essentially of finite type, $n:=\operatorname{dim} R \geq 4, I \subseteq R$ an ideal, height $I=n-2=$ $\operatorname{cd} I$. Assume that there exist $a, b \in R$ such that $(a, b) R$ is prime and $a, b$ define a system of parameters for $R / I$, let $p \in(a, b) R$ be a prime element. Then

$$
p R \in \operatorname{Ass}_{R} D\left(H_{I}^{n-2}(R)\right) .
$$

Proof. Obviously $R$ has only countably many prime ideals (as any algebra of finite type over $k$ has only countably many (prime) ideals). By [ $\underline{6}$, Theorem 2.1] there exist infinitely many prime ideals $q$ which contain $p$ and which are associated to $D\left(H_{I}^{n-2}(R)\right)$. For each such $q$ one has in particular $0 \neq \operatorname{Hom}_{R}\left(R / q, D\left(H_{I}^{n-2}(R)\right)\right) \stackrel{\left(*_{1}\right)}{=} D\left(H_{I}^{n-2}(R) \otimes_{R} R / q\right) \stackrel{\left(*_{2}\right)}{=} D\left(H_{I}^{n-2}(R / q)\right)$ $\left(\left(*_{1}\right)\right.$ : Hom-Tensor adjointness, $\left(*_{2}\right)$ : Right exactness of $\left.H_{I}^{n-2}\right)$ and hence height $(q) \leq 2$. As, therefore, all these $q$ have either height one (in which case $q$ equals $p R$ ) or height two, their intersection is $p R$ (the height of this intersection is necessarily one, as infinitely many pairwise different $q \mathrm{~s}$ are intersected). It follows that the intersection of all associated prime ideals of $\operatorname{Hom}_{R}\left(R / p R, D\left(H_{I}^{n-2}(R)\right)\right)$ is $p R$. By [8, Lemma 3.1], the associated prime ideals of $D\left(H_{I}^{n-2}(R)\right)$ are precisely the coassociated prime ideals of $H_{I}^{n-2}(R)$. [9, Folgerung 1.5 and Lemma 3.1] imply that each prime ideal minimal over $p R$ is associated to $H_{I}^{n-2}(R)$. But $p R$ is prime and hence we get $p R \in \operatorname{Coass}_{R}\left(H_{I}^{n-2}(R)\right)=\operatorname{Ass}_{R} D\left(H_{I}^{n-2}(R)\right)$.

Theorem 2.2. Let $k$ be a field, $X_{1}, \ldots, X_{n}$ indeterminates, $n \geq 4$. Set $R=k\left[\left[X_{1}, \ldots, X_{n}\right]\right]$ and $I=\left(X_{1}, \ldots, X_{n-2}\right) R$. Let $p \in\left(X_{n-1}, X_{n}\right) R$ be a prime element that has $p R \cap R_{0} \neq 0$, where $R_{0}:=k_{0}\left[X_{1}, \ldots, X_{n}\right]_{\left(X_{1}, \ldots, X_{n}\right)}$ and where $k_{0}$ is a countable subfield of $k$ (e. $g$. the prime subfield of $k$ ). Then

$$
p R \in \operatorname{Ass}_{R}\left(D\left(H_{I}^{n-2}(R)\right)\right) .
$$

Proof. $p R \cap R_{0}$ has height at most one, by our hypothesis it must hence have the form $p_{0} R_{0}$ for some prime element $p_{0} \in R_{0}$ (note that prime elements are non-zero by definition). As $k_{0}$ is countable, we get from theorem 2.1

$$
p_{0} R_{0} \in \operatorname{Ass}_{R_{0}}\left(D\left(H_{\left(X_{1}, \ldots, X_{n-2}\right) R_{0}}^{n-2}\left(R_{0}\right)\right)\right)
$$

(here $D$ is taken with respect to $R_{0}$, of course). By [8, Lemma 3.1], $p_{0} R_{0} \in$ $\operatorname{Coass}_{R_{0}}(\underbrace{H_{\left(X_{1}, \ldots, X_{n-2}\right) R_{0}}^{n-2}\left(R_{0}\right)}_{=: H})$. That means there exists an Artinian quotient $H \rightarrow H / B$ of $H$ that has

$$
\operatorname{Ann}_{R_{0}}(H / B)=p_{0} R_{0} .
$$


The $R$-module

$$
(H / B) \otimes_{R_{0}} R \stackrel{R / R_{0} \text { faithfully flat }}{=}\left(H \otimes_{R_{0}} R\right) /\left(B \otimes_{R_{0}} R\right)
$$

is a quotient of $H \otimes_{R_{0}} R$ and is Artinian (because its support is zerodimensional and its socle

$$
\operatorname{Hom}_{R}\left(R / m,(H / B) \otimes_{R_{0}} R\right)=\operatorname{Hom}_{R_{0}}\left(R_{0} /\left(X_{1}, \ldots, X_{n}\right), H / B\right) \otimes_{R_{0}} R
$$

has finite vector space-dimension); furthermore, by faithful flatness of $R / R_{0}$, its annihilator is

$$
\operatorname{Ann}_{R}\left((H / B) \otimes_{R_{0}} R\right)=p_{0} R .
$$

By Matlis duality, $D\left((H / B) \otimes_{R_{0}} R\right)$ is a finitely generated $R$-submodule of $D\left(H_{I}^{n-2}(R)\right)$ with annihilator

$$
\operatorname{Ann}_{R}\left(D\left((H / B) \otimes_{R_{0}} R\right)\right)=\operatorname{Ann}_{R}\left((H / B) \otimes_{R_{0}} R\right)=p_{0} R .
$$

The prime ideal $p R$ is minimal over $p_{0} R$, therefore we get

$$
p R \in \operatorname{Ass}_{R}\left(D\left((H / B) \otimes_{R_{0}} R\right)\right) \subseteq D\left(H_{I}^{n-2}(R)\right) .
$$

Remark 2.3. - In the situation of theorem 2.2 one can quickly show that $\{0\} \in \operatorname{Ass}_{R} D\left(H_{I}^{n-2}(R)\right)$ using the following arguments (this case was already known, with a different proof, see [1, Lemma 2.1.1]): The intersection of all coassociated prime ideals of $H_{I}^{n-2}(R)$ equals the radical of $\operatorname{Ann}_{R} H_{I}^{n-2}(R)$ (this follows from [9, Satz 1.2 and Folgerung 1.3], because $0=H_{I}^{n-2}\left(R /\left(X_{1}, \ldots, X_{n}\right)\right)=H_{I}^{n-2}(R) \otimes_{R} R /\left(X_{1}, \ldots, X_{n}\right) R$, $i$. e. one has $\left.\left(X_{1}, \ldots, X_{n}\right) H_{I}^{n-2}(R)=H_{I}^{n-2}(R)\right)$; but the endomorphism ring of $H_{I}^{n-2}(R)$ is $R$, by [4, Theorem 2.2 (iii)]; in particular, $\operatorname{Ann}_{R} H_{I}^{n-2}(R)=0$. Therefore, using the argument from the proof of theorem [2.1, one concludes $\{0\} \in \operatorname{Ass}_{R}\left(D\left(H_{I}^{n-2}(R)\right)\right)$.

It seems natural to ask

Question 2.4. In the situation of theorem 2.2, is it true that

$$
p R \in \operatorname{Ass}_{R}\left(D\left(H_{I}^{n-2}(R)\right)\right)
$$

holds for every prime element $p \in\left(X_{n-1}, X_{n}\right) R$ ?

Question 2.5. Does conjecture $(*)$ hold in this context, $i$. e. is it true that

$$
\operatorname{Ass}_{R}\left(D\left(H_{I}^{n-2}(R)\right)\right)=\left\{p \in \operatorname{Spec} R \mid H_{I}^{n-2}(R / p) \neq 0\right\} ?
$$

With respect to prime ideals of height two or zero both questions have positive answer, this was explained in the introduction. The results in this paper say that both questions have at least often a positive answer for height one prime ideals. 


\section{Non-finiteness properties}

Whenever, over a local, complete ring $(R, m)$, a given local cohomology module $H$ has infinitely many coassociated prime ideals (this is often the case: [2, Theorem 3.1.3 (ii), (iii)]), $H$ is neither finitely generated (because if it was, then $D(H)$ would be Artinian and hence one would have $\operatorname{Ass}_{R} D H=$ $\{m\})$ nor Artinian (because if it was then $\operatorname{Ass}_{R}(D(H)$ ) would be finite). This trivial remark is generalized.

Remark 3.1. Over the noetherian ring $R$, the coatomic modules are closed under taking quotients, submodules and extensions, see [7, section 1]. It is clear that every finitely generated $R$-module is coatomic and that every coatomic, Artinian module has finite length. Furthermore, localizations of coatomic modules are coatomic (over the localized ring), see [7, section 1, Folgerung 2].

Theorem 3.2. Let $R$ be a noetherian ring, $M$ an $R$-module and $I$ an ideal of $R$ such that $1 \leq c:=\operatorname{cd}(I, M)=\operatorname{cd}\left(I, R / \operatorname{Ann}_{R}(M)\right)<\infty$ (without further assumption one would have only $\operatorname{cd}(I, M) \leq \operatorname{cd}\left(I, R / \operatorname{Ann}_{R}(M)\right)$ in general). Then the top local cohomology module $H_{I}^{c}(M)$ has no non-zero coatomic quotient.

Proof. If $H_{I}^{c}(M)$ had a non-zero, coatomic quotient $H_{I}^{c}(M) / U$, then, by localizing in an arbitrary $p \in \operatorname{Supp}_{R}\left(H_{I}^{c}(M) / U\right)$, we would get a non-zero, coatomic (remark 3.1) quotient of $H_{I}^{c}(M)_{p}=H_{I R_{p}}^{c}\left(M_{p}\right)$. Therefore, we may replace $R$ by $R_{p}$ and assume that $(R, m)$ is local (note also that one has $\left.c=\operatorname{cd}(I, M)=\operatorname{cd}\left(I R_{p}, M_{p}\right)\right)$.

Assume to the contrary that $H / U$ is a non-zero, coatomic quotient of $H:=H_{I}^{c}(M)$ for some submodule $U$ of $H$. In particular there exists a maximal submodule $U^{\prime}$ of $H$ containing $U$. Being a simple module, $H / U^{\prime}$ is ismomorphic to $R / m$.

On the other hand, $D\left(H / U^{\prime}\right)$ is naturally a submodule of $D(H)$ and it is also isomorphic to $R / m$. But $m$ is not associated to $D\left(H / U^{\prime}\right) \subseteq D(H)$ (because otherwise

$$
0 \neq \operatorname{Hom}_{R}(R / m, D(H))=D\left(H_{I}^{c}(M) \otimes_{R}(R / m)\right) \stackrel{(\dagger)}{=} D\left(H_{I}^{c}(M / m M)\right)=0,
$$

contradiction; for $(\dagger)$ one works over the ring $R / \operatorname{Ann}_{R}(M)$ and uses the fact that $H_{I\left(R / \operatorname{Ann}_{R}(M)\right)}^{c}$ is right exact on $R / \operatorname{Ann}_{R}(M)$-modules). Therefore, no such quotient $H / U$ exists and the theorem is proven.

Note that in the formulation of theorem 3.2 (as well as in the subsequent corollary 3.3) it is not required that $M$ is finitely generated.

Corollary 3.3. Let $I$ be an ideal of a noetherian ring and let $M$ be an $R$ module such that $1 \leq c:=\operatorname{cd}(I, M)=\operatorname{cd}\left(I, R / \operatorname{Ann}_{R}(M)\right)<\infty$. Then $H_{I}^{c}(M)$ has no non-zero finitely generated quotient. 
Remark 3.4. The proof of the preceding theorem actually shows that in the given situation the top local cohomology module is radikalvoll (see e. g. [7] for this terminology: By definition, a module is radikalvoll if it has no maximal submodule).

As an application of theorem 3.2 we get immediately an improvement of [2, Cor. 1.1.4] (recall that a sequence $\left(x_{1}, \ldots, x_{n}\right)$ in a local ring $R$ is filter regular on the $R$-module $M$ if, for each $i$, the kernel of the multiplication map $M /\left(x_{1}, \ldots, x_{i-1}\right) M \stackrel{x_{i}}{\rightarrow} M /\left(x_{1}, \ldots, x_{i-1}\right) M$ is Artinian) see e. g. [10] and [11]):

Theorem 3.5. Let $(R, m)$ be a noetherian, local ring, $I$ a proper ideal of $R, h \in \mathbb{N}$ and $\underline{f}=f_{1}, \ldots, f_{h} \in I$ an $R$-regular sequence. The following statements are equivalent:

1. $\sqrt{\underline{f} R}=\sqrt{I}$.

2. $H_{I}^{l}(R)=0$ for every $l>h$ and the sequence $\underline{f}$ is quasi-regular on $D\left(H_{I}^{h}(R)\right)$.

3. $H_{I}^{l}(R)=0$ for every $l>h$ and the sequence $\underline{f}$ is regular on $D\left(H_{I}^{h}(R)\right)$.

4. $H_{I}^{l}(R)=0$ for every $l>h$ and the sequence $\underline{f}$ is filter regular on $D\left(H_{I}^{h}(R)\right)$.

Proof. Because of [2, Cor. 1.1.4] it suffices to show that 4, implies 2, Assume that $h \geq 1, H_{I}^{l}(R)=0$ for every $l>h$ and that $\underline{f}=f_{1}, \ldots, f_{h} \in I$ is a filter regular sequence on $D\left(H_{I}^{h}(R)\right)$. In particular, the kernel $K$ of the multiplication map

$$
D\left(H_{I}^{h}(R)\right) \stackrel{f_{1}}{\rightarrow} D\left(H_{I}^{h}(R)\right)
$$

is Artinian. But $K=\operatorname{Hom}_{R}\left(R / f_{1}, D\left(H_{I}^{h}(R)\right)\right)=D\left(H_{I}^{h}(R) \otimes_{R}\left(R / f_{1} R\right)\right)$ and hence the quotient module $H_{I}^{h}(R) \otimes_{R}\left(R / f_{1}\right)$ is a finitely generated $\hat{R}$-module. It follows from theorem 3.2 that $K=0$. But then we have $\left.D\left(H_{I}^{h-1}\left(R / f_{1} R\right)\right)=D\left(H_{I}^{h}(R)\right) \otimes_{R}\left(R / f_{1}\right) R\right)$ by an easy argument with exact sequences. Now it is clear that the claim follows by induction on $h$.

\section{References}

[1] Hellus, M. On the associated primes of Matlis duals of top local cohomology modules, Communications in Algebra 33 (2005), no. 11, 3997-4009.

[2] Hellus, M. Local Cohomology and Matlis Duality, Habilitationsschrift, Leipzig, 2006, available from http://www.math.uni-leipzig.de/ hellus/HabilitationsschriftOhneDeckblatt.pdf 
[3] Hellus, M. A note on the injective dimension of local cohomology modules, Proceedings of the American Mathematical Society 136 (2008), 2313-2321.

[4] Hellus, M. and Stückrad, J. On endomorphism rings of local cohomology modules, Proceedings of the American Mathematical Society 136 (2008), 2333-2341.

[5] Hellus, M. and Stückrad, J. Matlis duals of top Local Cohomology Modules, Proceedings of the American Mathematical Society 136 (2008), 489498.

[6] Hellus, M. and Stückrad, J. Artinianness of local cohomology, to appear in the Journal of Commutative Algebra.

[7] Zöschinger, H. Koatomare Moduln, Math. Z. 170 (1980), 221 - 232.

[8] Zöschinger, H. Minimax-Moduln, J. Algebra 102 (1986), 1-32.

[9] Zöschinger, H. Über koassoziierte Primideale, Math. Scand. 63 (1988), 196-211.

[10] Schenzel, P., Trung, N. V. and Cuong, N. T., Verallgemeinerte CohenMacaulay-Moduln, Math. Nachr. 85 (1978), 57-73.

[11] Stückrad, J. and Vogel, W., Buchsbaum rings and Applications, VEB Deutscher Verlag der Wissenschaften, Berlin (1986).

Michael Hellus, Universität Leipzig, Fakultät für Mathematik und Informatik, PF 1009 20, 04009 Leipzig, Germany

E-Mail: hellus@math.uni-leipzig.de 